\title{
Riesgo de exposición a Fasciola hepatica en ganado vacuno en extensivo de Uruguay y Portugal determinado mediante ELISA y un antígeno recombinante
}

\author{
Risk of exposure to Fasciola hepatica in cattle under extensive management from Uruguay \\ and Portugal determined by ELISA using a recombinant antigen \\ J Sanchís ${ }^{\text {ab }}$, GV Hillyer ${ }^{\mathrm{c}}$, LM Madeira de Carvalho ${ }^{\mathrm{d}}$, MI Macchi ${ }^{\mathrm{b}}$, C Gomes $^{\mathrm{d}}$, G Maldini ${ }^{\mathrm{b}}$, G Stilwell ${ }^{\mathrm{d}}$, \\ JM Venzal ${ }^{\mathrm{b}}$, A Paz-Silva ${ }^{\mathrm{a}}$, R Sánchez-Andrade ${ }^{\mathrm{a}}$, MS Arias ${ }^{\mathrm{a}}{ }^{*}$ \\ aDepartamento Patología Animal, Epidemiología, Zoonosis y Enfermedades Parasitarias, Facultad de Veterinaria, Universidad de \\ Santiago de Compostela, Lugo, España. \\ bParasitología, Universidad de la República, Salto, Uruguay. \\ 'Laboratorio de Inmunología Parasitaria y Patología, Escuela de Medicina, Campus de Ciencias Médicas, Universidad de Puerto \\ Rico, San Juan, Puerto Rico. \\ ${ }^{\mathrm{d} C I I S A}$, Facultad de Medicina Veterinaria, Universidad Técnica de Lisboa, Lisboa, Portugal.
}

\section{SUMMARY}

\begin{abstract}
The risk of exposure to Fasciola hepatica in cattle maintained under extensive conditions from two different climatic areas was evaluated. Bovine serum samples collected in Uruguay (humid subtropical climate; 1,192 samples) and Portugal (mediterranean climate; 473 samples) were tested against a F. hepatica recombinant surface antigen (FhrAPS) by means of an ELISA. Data obtained was analyzed according to the bovine breed, age and aptitude. An overall percentage of 56\% (95\% CI, 53-59) cattle were positive to the FhrAPS-ELISA in Uruguay, with significantly highest percentages in 2-4 year Holstein dairy cows. In Portugal, the percentage of sensitization was 47\% (95\% CI, 43-52) with the highest values recorded among Crossbreeds and beef bovines between 2-4 years of age. By means of the FhrAPS-ELISA, it was concluded the risk of exposure to F. hepatica among extensively reared cattle is higher in areas under humid subtropical climate than in those with Mediterranean climate. Calves are at higher risk of being exposed to the liver trematode independent of the climate conditions which emphasises the need for preventive measures against infective stages found in the environment and combne them with successful deworming programs.
\end{abstract}

Key words: cattle fasciolosis, grazing, risk assessment, immunoassay.

\section{RESUMEN}

Para evaluar el riesgo de exposición a Fasciola hepatica del ganado vacuno en extensivo se tomaron muestras de sangre de dos zonas climáticas diferentes, Uruguay (clima subtropical húmedo, 1.192 muestras) y Portugal (clima mediterráneo, 473 muestras), que se analizaron con un ELISA y un antígeno recombinante de superficie de $F$. hepatica (FhrAPS). Los datos obtenidos se analizaron de acuerdo con la raza, edad sexo y aptitud. La seroprevalencia global en Uruguay resultó del 56\% (Intervalo de Confianza, IC 95\%: 53-59) mediante FhrAPS-ELISA, con porcentajes significativamente más altos en vacas lecheras Holstein de 2-4 años. En Portugal, el porcentaje de sensibilización fue del 47\% (IC 95\%: 43-52) con los valores más elevados en bovinos resultado de cruces de distintas razas, destinados a la obtención de carne y con edad también comprendida entre 2 y 4 años. Se concluyó que el riesgo de exposición a $F$. hepatica del ganado vacuno en extensivo es mayor en las zonas con clima subtropical húmedo que en las de clima mediterráneo. Los terneros presentan un alto riesgo de exposición al trematodo hepático con independencia de las condiciones climáticas. Los resultados ponen de manifiesto la necesidad de adoptar medidas frente a las formas del parásito que se encuentran en el medio y combinarlas con programas de desparasitación eficaces.

Palabras clave: fasciolosis, pastoreo, riesgo de exposición, inmunoensayo.

\section{INTRODUCCIÓN}

El desarrollo del ciclo de F. hepatica depende de varios factores (temperatura, humedad, presencia de hospedadores intermediarios adecuados, etc.) y en consecuencia, la prevalencia de infección de los hospedadores definitivos

Aceptado: 20.08.2014.

* Pabellón 1, Planta Baja, Avda. Carvallo Calero, s/n, 27002 Lugo, España; mariasol.arias@usc.es varía sensiblemente de unas zonas a otras. También influyen las condiciones orográficas, como el tipo de suelo, la pendiente, etc. (Rojo y Ferre 1999). En este sentido, en los últimos 60 años, el hombre ha realizado cambios en el medio, mediante la construcción de presas, diversos sistemas de irrigación, etc., de modo que ha transformado lugares secos en húmedos, lo que ha contribuido notablemente a la expansión geográfica de la fasciolosis (Otranto y Traversa 2003).

Los animales parasitados por $F$. hepatica eliminan huevos en las heces, estos, al caer en zonas encharcadas 
con temperaturas superiores a $10{ }^{\circ} \mathrm{C}$, desarrollan a los pocos días en su interior un miracidio, embrión capaz de infectar a los hospedadores intermediarios, caracoles anfibios limneidos (Bennema y col 2011). Cuando la condición ambiental, en particular la temperatura, no es la óptima la evolución es retardada llegando incluso a ser inhibida completamente a una temperatura inferior a $10{ }^{\circ} \mathrm{C}$. Por lo anterior el ciclo queda interrumpido en el periodo de otoño-invierno donde no se producen nuevas infecciones (Alcaíno y Apt 1989). Después de completar varias fases se forman las cercarias, que abandonan los caracoles y se transforman en metacercarias en el ambiente (fase infectiva). De esta manera, el riesgo de exposición al trematodo hepático guarda relación directa con las condiciones climáticas que determinan la supervivencia de los caracoles y metacercarias en el medio ambiente (Dunkel y col 1996).

La cría de ganado en extensivo, en la que los animales no se guarecen por la noche y obtienen su alimento mediante el pastoreo y a campo abierto, siendo el ganado pocas veces suplementado aumenta su exposición a algunos agentes patógenos como Fasciola hepatica, responsable de importantes pérdidas económicas por anemia, disminución de la producción de leche y decomiso del hígado (Reid y Dargie 1995). Se han detectado reducciones en la ganancia de peso vivo de terneros entre 0,07 y $1,2 \mathrm{~kg}$ / semana (reducción total del 4,1 al 28\%) (Marley y col 1996, Schweizer y col 2005, Sariözkan y Yalçin 2011). Las novillas con fasciolosis requieren más inseminaciones que las no infectadas (Hope Cawdery 1984).

Asimismo, la explotación del ganado en extensivo es posible en las regiones donde el forraje verde crece durante la mayor parte del año. En estos territorios, donde los climas son mesotermales (con una temperatura media superior a $10^{\circ} \mathrm{C}$ en los meses más cálidos), este tipo de manejo está muy extendido.

Entre los climas mesotermales, el húmedo subtropical se caracteriza por veranos calurosos húmedos e inviernos suaves (Kottek y col 2006). Las precipitaciones son constantes a lo largo del año, por lo que no existe un periodo seco. Estas condiciones favorecen la cría del ganado en condiciones naturales (Carámbula 1996). Este tipo de clima sucede en el lado sureste de todos los continentes, por lo general entre las latitudes $25^{\circ}$ y $40^{\circ}$ norte, comprendiendo áreas del sureste de Asia, África, Medio Oriente, América del Norte y del Sur, y este de Australia.

El clima mediterráneo (también clasificado como mesotermal) se define por veranos calurosos y secos e inviernos lluviosos y fríos, momentos en los que se reduce el crecimiento del forraje. Las condiciones climáticas adversas y la escasez de pasto en invierno obligan a la estabulación del ganado, época en la que se alimenta con forraje seco o ensilado y concentrado (pienso). Este clima por lo general se da en los lados occidentales de los continentes, entre las latitudes de $30^{\circ} \mathrm{y} 45^{\circ}$, por lo que se puede encontrar en el sur de Europa, California, suroeste de Sudáfrica, Australia Occidental y del Sur.

El tipo de explotación extensiva proporciona ventajas frente a la ganadería intensiva, fundamentalmente menos mano de obra, mecanización de labores agrícolas y mejora el bienestar animal. El ganado mantenido en pastoreo proporciona alimentos de mejor calidad, como carne que se considera natural certificada (con pasto y libre de hormonas) o carne etiquetada con certificación de alimento orgánico.

Los métodos más utilizados para el diagnóstico directo de las infecciones por $F$. hepatica han sido los exámenes coprológicos y la necropsia (Rojo y Ferre 1999). Debido al alto valor económico de estos animales, los datos de necropsia se reducen a trabajos puntuales que se han realizado en matadero. La técnica coprológica de sedimentación se considera que es un método de diagnóstico tardío, permite el diagnóstico directo de la fasciolosis por la visualización de los huevos en las muestras fecales, pero solo proporciona resultados útiles después del período de prepatencia, cuando la mayoría de las lesiones patológicas ya se han producido (Carnevale y col 2001). Los análisis coprológicos son de gran utilidad debido a su alta especificidad y a su sencillez. No obstante, diversos autores señalan que es necesario realizar varios análisis antes de pronunciarse si un animal está o no infectado por $F$. hepatica y que como mínimo deberían realizarse 2 o 3 coprologías, realizadas con intervalos de algunos días e incluso semanas; además, se ha comprobado que, cuando los análisis coprológicos se realizan una sola vez, puede haber hasta un 65\% de falsos negativos (Chauvin 1995).

Para la detección de las infecciones por $F$. hepatica durante las primeras etapas se han utilizado diferentes métodos de inmunodiagnóstico, orientados a la detección de anticuerpos específicos ( $\mathrm{IgG}$ ) frente a antígenos del trematodo. Entre estas técnicas, la más utilizada ha sido el ELISA (Paz-Silva y col 2003) utilizando antígeno de excreción/secreción de $F$. hepatica, que permite realizar un diagnóstico temprano de la infección. Aunque el ELISA presenta gran sensibilidad, especificidad y fácil reproductibilidad (Oldham 1982, Ibarra y col 1998), la persistencia de una elevada tasa de anticuerpos durante largos periodos, incluso cuando los animales han sido capaces de eliminar de forma natural la infección o tras la administración de un tratamiento eficaz, supone un inconveniente importante (Sánchez-Andrade y col 2000). Con objeto de superar estos inconvenientes, se busca la producción de antígenos unitarios, mediante la obtención y purificación de proteínas recombinantes (Carnevale y col 2001) que permitan diferenciar animales con infección activa de los que la han superado.

Se han desarrollado diferentes pruebas serológicas para el diagnóstico de la fasciolosis bovina (Cornelissen y col 2001, Rapsch y col 2006, Salimi-Bejestani y col 2008, Mezo y col 2011, Sen y col 2011). Los ensayos inmunoenzimáticos permiten evaluar la exposición de los 
animales al trematodo (Sánchez-Andrade y col 2000, Arias y col 2010), lo que resulta útil para obtener información sobre la distribución de la infección, las zonas de riesgo y otros posibles factores implicados (Kuerpick y col 2012).

Con objeto de determinar si el clima influye en la posibilidad de exposición a $F$. hepatica del ganado vacuno en extensivo, en la presente investigación se obtuvieron sueros de bovinos de dos países con diferentes características climáticas, Uruguay (clima subtropical húmedo) y Portugal (clima mediterráneo). La presencia de anticuerpos IgG frente al trematodo se evaluó mediante un antígeno recombinante de superficie de $F$. hepatica de 2,9 kDa (FhrAPS).

\section{MATERIAL Y MÉTODOS}

\section{ÁREAS DE ESTUDIO}

Entre agosto y diciembre de 2011 se recogieron muestras de sangre de 1.192 bovinos pertenecientes a 40 fincas en Uruguay ( $33^{\circ} 00^{\prime} 00^{\prime \prime} \mathrm{S}, 56^{\circ} 00^{\prime} 00^{\prime \prime} \mathrm{W}$ ). Por su situación en la zona templada del hemisferio sur tiene un clima moderado, con valores máximos de temperatura en enero y mínimos en julio, y una pequeña variación térmica anual (alrededor de $8^{\circ} \mathrm{C}$ ) (Fox y col 2011, Eleftheratos y col 2011). Las precipitaciones son abundantes y regulares en cualquier época del año (332 mm en primavera, 360 en verano, 407,8 en otoño y 277,9 en invierno), siendo designado por Köppen como clima Cfa (Kottek y col 2006).

Desde marzo de 2011 a febrero de 2012 se tomaron muestras de sangre de un total de 473 bovinos en 17 granjas de Portugal ( $39^{\circ} 30^{\prime} 00^{\prime \prime} \mathrm{N}, 8^{\circ} 00^{\prime} 00^{\prime}$ W). El clima se caracteriza por tener veranos calurosos y secos e inviernos fríos y lluviosos, en las zonas costeras las temperaturas tanto en el verano como en el invierno son más suaves por la presencia cercana del mar (Kuchai y col 2011). La precipitación media anual es de $86,7 \mathrm{~mm}$ en primavera, $38,7 \mathrm{~mm}$ en verano, $163,5 \mathrm{~mm}$ en otoño y $129,3 \mathrm{~mm}$ en invierno, siendo denominado por Köppen como clima Csa (Kottek y col 2006).

\section{GANADO VACUNO}

Uruguay. El censo bovino en Uruguay es de alrededor de 11.805.573 animales destinados a la producción de carne (94\%) y de leche (5\%). Las vacas de razas cárnicas (Hereford, Angus y cruces) se mantienen todo el año en pastizales naturales compuestos por una asociación de Paspalum notatum (pasto horqueta) y Axonopus affinis (pasto chato) que contribuye con más del $30 \%$ de la materia seca total. Otras especies presentes en orden decreciente son: ciperáceas, Coelorhachis selloana (cola de lagarto), Paspalum dilatatum (pasto miel), Stenotaphrum secundatum (gramillón), Panicum milioides (pasto tierno), Cynodon dactylon (gramilla), Setaria geniculata (pasto amargo) y Axonopus argentinus (pasto chato). El 77\% de la producción de forraje se consigue a partir de 10 especies. Durante el invierno, los animales pueden pastorear raigrás (Lolium perenne, L. multiflorum) y avena (Avena sativa). La rotación de los pastos está en función de la abundancia del forraje, y si es necesario los animales se suplementan con ensilado de sorgo (Sorghum bicolor) o concentrado. Disponen de agua procedente de pozos naturales, cañadas o arroyos.

Los bovinos de leche (Holstein), a diferencia del vacuno de carne, aprovechan praderas húmedas durante todo el año, en otoño, invierno y primavera pastan trébol (Trifolium spp.), Lotus (Lotus corniculatus), avena y raigrás, y en verano praderas de sorgo. En algunas praderas también se cultiva trigo (Triticum aestivum), festuca (Festuca arundinacea) y pasto ovillo (Dactylis glomerata). La alimentación del vacuno lechero se complementa con ensilado de sorgo o maíz (Zea mays). Las vacas Holstein se ordeñan 1-2 veces/ día, momento en el que ingieren agua de bebederos con agua corriente.

El control de la fasciolosis consiste en la administración de nitroxinil, closantel o triclabendazol. El ganado de carne (novillas principalmente) recibe dos dosis, al comienzo del otoño y en la primavera. Las vacas lecheras se desparasitan durante el período de secado.

Portugal. Existe un censo aproximado de 1.529.000 reses de vacuno en Portugal (INE 2012), de las que aproximadamente el 35\% se mantiene en régimen extensivo (Limousine, cruces y Charolais) para recría o producción de carne. Se trata de rumiantes que se alimentan en pastizales naturales (compuestos por Erica arborea, Poa pratensis, Dactylis glomerata, Phleum pratense y Chamomilla recutita) o cultivados (Trifolium pratense, T. repens, Lolium multiflorum). En todos los casos, cuando el forraje es escaso (diciembre a febrero), se les proporciona suplementación a base de concentrado (pienso).

Los animales solo se estabulan si las condiciones climáticas son muy desfavorables.

El control parasitario consiste en la administración de antiparasitarios (albendazol, ivermectina más clorsulón, ivermectina más closantel o solo closantel) una vez al año.

Las vacas de leche (Holstein) suelen permanecer estabuladas durante el período de ordeño, y solo pastan en el período seco, por lo que no se tuvieron en cuenta en el estudio actual.

\section{MUESTREO}

La toma de muestras de sangre se realizó con el apoyo de veterinarios clínicos que habitualmente colaboran con la Universidad de la República (Uruguay) y la Universidad Técnica de Lisboa (Portugal). Ninguno de los propietarios se negó a colaborar.

Teniendo en cuenta que en ambos países las vacas lecheras generalmente se sacrifican a los 7-9 años, solo se incluyeron en el presente estudio bovinos menores de 9 
años. En función de la edad se establecieron tres grupos: $<2$ años, 2-4 años y > 4 años.

\section{PROTOCOLO FHRAPS-ELISA}

La presencia de anticuerpos séricos frente al antígeno de superficie FhrAPS se evaluó en el Laboratorio de Enfermedades Parasitarias (Universidad de Santiago de Compostela, España) mediante un ELISA (Paz-Silva y col 2007), técnica que cuenta con una sensibilidad del $86 \%$ y especificidad del 88\% (Arias y col 2010).

Los sueros utilizados como testigos negativos se obtuvieron de 42 terneros estabulados desde su nacimiento que nunca eliminaron huevos del trematodo en las heces. La técnica copromicroscópica empleada para evidenciar la ausencia de huevos en las heces de estos animales fue la sedimentación (MAFF 1986). El punto de corte para un resultado positivo se tomó como la densidad óptica media (DO) de todos los sueros negativos más tres veces la desviación estándar (Sánchez-Andrade y col 2002). Por lo tanto, las muestras se consideraron positivas cuando los valores de DO fueron $>0,3887$.

\section{ANÁLISIS ESTADÍSTICO}

Los resultados se expresan como porcentajes de seroprevalencia verdadera (Greiner y Gardner 2000), el intervalo de confianza al 95\%, y se analizaron mediante la prueba de $\chi^{2}$. Las diferencias se consideraron significativas cuando $\mathrm{P}<0,05$.

Las posibles relaciones entre la seroprevalencia, la raza, edad o sexo, como variables independientes (predictores) se estimó mediante el cálculo de la razón de posibilidades (OR, odds ratio) de seropositividad (resultado) mediante regresión logística (Thrusfield 2005). Los análisis se realizaron utilizando el software IBM SPSS Statistics (20.0.0).

\section{LAS NORMAS ÉTICAS}

Esta investigación fue sometida a revisión ética y fue aprobada por el Comité de Ética de la Universidad de la República (Regional Norte), Salto (Uruguay) y de la Universidad Técnica de Lisboa (Portugal). En el manejo de los bovinos se respetaron las leyes de bienestar animal y las directrices de ambos países.

\section{RESULTADOS}

\section{SEROPREVALENCIA INDIVIDUAL}

La seroprevalencia de fasciolosis en Uruguay fue del $56 \%$ (IC $95 \%, 53-59$ ) y del 47\% (43-52) en Portugal (P $=0,001)$.

En Uruguay se alcanzaron porcentajes significativamente más altos de positividad en vacas de raza Holstein, y en Portugal en la raza Cruces (Cuadro 1). Respecto de la edad, las mayores seroprevalencias se observaron en los animales de 2-4 años de ambos países (Uruguay: 64\%, 60-68; Portugal: 61\%, 51-70). El porcentaje de bovinos menores de dos años que resultaron positivos al FhrAPSELISA, fue del 51\% (46-56) en Uruguay y del $43 \%$ (32-54) en Portugal $(P=0,179)$.

En cuanto a la aptitud, se demostraron seroprevalencias significativamente más elevadas en el ganado lechero de Uruguay, y en el de carne en Portugal.

El cuadro 1 resume que no se observaron diferencias estadísticamente significativas según el sexo, aunque se obtuvieron valores de seropositividad más altos en las vacas en Uruguay y los toros en Portugal.

\section{ANÁLISIS DE RIESGO}

El valor más elevado de OR se obtuvo entre las vacas Holstein (aptitud lechera) y en los animales de 2-4 años de edad en Uruguay (cuadro 1), y en los Cruces (carne) y en animales de 2-4 años de edad en Portugal.

\section{SEROPREVALENCIA EN EXPLOTACIONES}

El 95\% de las explotaciones del Uruguay y el $71 \%$ de las de Portugal tenían al menos un animal seropositivo. Todas las granjas de Uruguay, con mayoría de vacas Holstein o Hereford, tenían algún animal seropositivo y en Portugal las granjas con vacuno resultado del cruce de diferentes razas alcanzaron los niveles más altos de seropositividad (cuadro 2).

Según la edad de los bovinos, se observó la menor prevalencia de explotaciones positivas en aquellos con rumiantes menores de dos años. No se demostraron diferencias significativas en cuanto a la edad, sexo ni aptitud (cuadro 2).

\section{DISCUSIÓN}

En la presente investigación se evaluó el riesgo de exposición al trematodo $F$. hepatica en ganado vacuno en régimen extensivo de dos países con diferente clima (subtropical húmedo y Mediterráneo). Mediante un ELISA con un antígeno recombinante de superficie de $F$. hepatica de 2,9 kDa (FhrAPS) (Paz-Silva y col 2005) se comprobó que casi todas las granjas de Uruguay tenían rumiantes seropositivos, con una prevalencia individual del 56\%. En un estudio previo desarrollado con antígenos de excreción/ secreción de trematodos adultos se demostró una seroprevalencia individual del $67 \%$ en la región de Salto (noroeste de Uruguay) (Sanchís y col 2011). Asimismo, entre 2007 y 2008, en los mataderos de Uruguay se decomisó el 37,4\% de hígados de bovinos debido a las lesiones provocadas por F. hepatica (Castro y Brito 2008).

En Portugal se ha diagnosticado la fasciolosis bovina mediante el uso de pruebas coprológicas y en mataderos (Conceição y col 2002, Arias y col 2011). El presente 
Cuadro 1. Seroprevalencia de fasciolosis en ganado vacuno mantenido en régimen extensivo de Uruguay (clima húmedo subtropical) y Portugal (clima mediterráneo).

Seroprevalence of fasciolosis in cattle extensively managed from Uruguay (humid subtropical climate) and Portugal (mediterranean climate).

\begin{tabular}{|c|c|c|c|c|c|c|c|c|c|c|}
\hline \multirow{3}{*}{ Raza } & \multicolumn{5}{|c|}{ Uruguay $(\mathrm{N}=1.192)$} & \multicolumn{5}{|c|}{ Portugal (N=473) } \\
\hline & \multirow{2}{*}{$\mathrm{N}$} & \multicolumn{2}{|c|}{$\mathrm{PV}$} & \multicolumn{2}{|c|}{ OR } & \multirow{2}{*}{$\mathrm{N}$} & \multicolumn{2}{|c|}{$\mathrm{PV}$} & \multicolumn{2}{|c|}{ OR } \\
\hline & & $\%$ & IC 95\% & Valor & IC 95\% & & $\%$ & IC $95 \%$ & Valor & IC 95\% \\
\hline Angus & 328 & 48 & $43-53$ & 0,7 & $0,5-0,9$ & & & & & \\
\hline Hereford & 427 & 56 & $51-61$ & 1,0 & $0,8-1,3$ & & & & & \\
\hline Holstein & 289 & 68 & $64-73$ & 2,0 & $1,5-2,6$ & & & & & \\
\hline Cruces & 148 & 47 & $42-52$ & 0,7 & $0,5-1,0$ & 223 & 57 & $51-64$ & 2,2 & $1,5-3,1$ \\
\hline Limousine & & & & & & 250 & 38 & $32-44$ & 0,5 & $0,3-0,7$ \\
\hline Estadística & \multicolumn{5}{|c|}{$\chi^{2}=30,076, P=0,001$} & & & \multicolumn{3}{|c|}{$\chi^{2}=17,066, P=0,001$} \\
\hline \multicolumn{11}{|l|}{ Edad (años) } \\
\hline$\overline{<2}$ & 391 & 51 & $46-56$ & 0,8 & $0,6-1,0$ & 81 & 43 & $32-54$ & 0,8 & $0,5-1,3$ \\
\hline $2-4$ & 543 & 64 & $60-68$ & 1,9 & $1,5-2,3$ & 107 & 61 & $51-70$ & 2,0 & $1,3-3,1$ \\
\hline$>4$ & 258 & 45 & $39-51$ & 0,6 & $0,4-0,8$ & 285 & 44 & $38-49$ & 0,7 & $0,5-1,0$ \\
\hline Estadística & \multicolumn{5}{|c|}{$\chi^{2}=29,618, P=0,001$} & & & \multicolumn{3}{|c|}{$\chi^{2}=9,948, P=0,007$} \\
\hline \multicolumn{11}{|l|}{ Sexo } \\
\hline Vaca & 964 & 56 & $53-60$ & & & 381 & 47 & $42-52$ & & \\
\hline Toro & 228 & 53 & $46-59$ & & & 92 & 49 & $39-59$ & & \\
\hline Estadística & \multicolumn{5}{|c|}{$\chi^{2}=0,639, P=0,424$} & & & \multicolumn{3}{|c|}{$\chi^{2}=0,111, \mathrm{P}=0,739$} \\
\hline \multicolumn{11}{|l|}{ Aptitud } \\
\hline Lechera & 289 & 68 & 63-74 & 2,0 & $1,5-2,6$ & & & & & \\
\hline Cárnica & 903 & 52 & $48-55$ & 0,5 & $0,4-0,7$ & 252 & 57 & $51-63$ & 2,3 & $1,6-3,3$ \\
\hline Cría & & & & & & 221 & 37 & $30-43$ & 0,4 & $0,3-0,6$ \\
\hline Estadística & \multicolumn{5}{|c|}{$\chi^{2}=23,385, P=0,001$} & & & \multicolumn{3}{|c|}{$\chi^{2}=19,070, P=0,001$} \\
\hline
\end{tabular}

Los resultados se expresan como porcentajes y el intervalo de confianza al 95\% (IC 95\%). Prevalencia verdadera (PV). Razón de posibilidades (OR: odds ratio).

trabajo mostró que el $71 \%$ de las granjas tenían bovinos con anticuerpos frente al trematodo hepático, con una exposición individual de los animales del 47\%. Estos valores son similares a los señalados previamente en el centro-norte de Portugal (Conceição y col 2004), y más bajos que los encontrados en una región endémica fronteriza del noroeste de España (Arias y col 2010).

Las condiciones climáticas influyen en la actividad de los hospedadores intermediarios de F. hepatica, y como consecuencia en la transformación de cercaria a metacercaria (fase infectiva) (Fox y col 2011). En zonas con clima subtropical húmedo parecen concurrir condiciones que favorecen la presencia de caracoles limneidos todo el año (Nari y col 1986), por lo que la seroprevalencia más alta en Uruguay podría atribuirse a una mayor contaminación de los pastos con metacercarias de F. hepatica. Sin embargo, las bajas temperaturas que se registran durante el invierno en las zonas de clima mediterráneo inducen a los caracoles a enterrarse en el barro (OMS 1997), reduciéndose de este modo la emisión de cercarias al medio. Como consecuencia, la infección de los vacas dependerá sobre todo del final de la hibernación de los caracoles infectados, porque a pesar de la resistencia de las metacercarias al frío, menos del $30 \%$ metacercarias sobreviven viables en el pasto hasta la primavera en regiones con clima mediterráneo (LuzónPeña y col 1995, Dunkel y col 1996).

La observación de las mayores seroprevalencias en los bovinos de 2-4 años de ambos países coincide con investigaciones previas en regiones endémicas, en las que utilizando un ELISA con antígeno recombinante (FhrAPS) se comprobó la exposición al trematodo hepático en más del $40 \%$ de los terneros (Sánchez-Andrade y col 2000, 2002).

Las condiciones climáticas afectan a la supervivencia y actividad de los hospedadores intermediarios de F. hepatica y a la viabilidad de las metacercarias, y condicionan el manejo del ganado, influyendo todos estos factores en el riesgo de exposición al trematodo. La observación de la mayor seroprevalencia en Uruguay entre las vacas Holstein confirma los resultados previamente obtenidos (Sanchís y col 2011), en animales que se alimentan en pastizales húmedos durante todo el año, lo que sin duda favorece la ingestión de metacercarias. Otro aspecto a 
Cuadro 2. Seroprevalencia de fasciolosis en explotaciones de ganado vacuno mantenido en régimen extensivo de Uruguay (clima húmedo subtropical) y Portugal (clima mediterráneo).

Farm-seroprevalence of fasciolosis in cattle extensively managed from Uruguay (humid subtropical climate) and Portugal (mediterranean climate).

\begin{tabular}{|c|c|c|c|c|c|c|c|c|}
\hline \multirow{3}{*}{ Raza } & \multicolumn{4}{|c|}{ Uruguay $(\mathrm{N}=40)$} & \multicolumn{4}{|c|}{ Portugal $(\mathrm{N}=17)$} \\
\hline & \multirow{2}{*}{$\mathrm{N}$} & \multicolumn{2}{|c|}{ PV } & \multirow{2}{*}{ Estadística } & \multirow{2}{*}{$\mathrm{N}$} & \multicolumn{2}{|c|}{ PV } & \multirow{2}{*}{ Estadística } \\
\hline & & $\%$ & IC $95 \%$ & & & $\%$ & IC $95 \%$ & \\
\hline$\overline{\text { Angus }}$ & 10 & 90 & $71-100$ & \multirow{5}{*}{$\begin{array}{l}\chi^{2}=3,509 \\
\mathrm{P}=0,320\end{array}$} & & & & \multirow{5}{*}{$\begin{array}{c}\chi^{2}=0,476 \\
\mathrm{P}=0,490\end{array}$} \\
\hline Hereford & 15 & 100 & & & & & & \\
\hline Holstein & 9 & 100 & & & & & & \\
\hline Cruces & 6 & 83 & $54-100$ & & 9 & 78 & $51-100$ & \\
\hline Limousine & & & & & 8 & 62 & $29-96$ & \\
\hline \multicolumn{9}{|l|}{ Edad (años) } \\
\hline$<2$ & 15 & 87 & $69-100$ & \multirow{3}{*}{$\begin{array}{l}\chi^{2}=3,509 \\
P=0,173\end{array}$} & 7 & 57 & $20-94$ & \multirow{3}{*}{$\begin{array}{c}\chi^{2}=1,862 \\
\mathrm{P}=0,394\end{array}$} \\
\hline $2-4$ & 8 & 100 & & & 7 & 71 & $38-100$ & \\
\hline$>4$ & 17 & 100 & & & 3 & 100 & & \\
\hline \multicolumn{9}{|l|}{$\overline{\text { Sexo }}$} \\
\hline$\overline{\text { Vaca }}$ & 34 & 97 & $91-100$ & \multirow{2}{*}{$\begin{array}{l}\chi^{2}=2,023 \\
P=0,155\end{array}$} & 11 & 73 & $46-99$ & \multirow{2}{*}{$\begin{array}{l}\chi^{2}=0,069 \\
P=0,793\end{array}$} \\
\hline Toro & 6 & 83 & $54-100$ & & 6 & 67 & $29-100$ & \\
\hline \multicolumn{9}{|l|}{ Aptitud } \\
\hline Lechera & 9 & 100 & & \multirow{3}{*}{$\begin{array}{l}\chi^{2}=0,611 \\
P=0,434\end{array}$} & & & & \multirow{3}{*}{$\begin{array}{l}\chi^{2}=0,383 \\
\mathrm{P}=0,536\end{array}$} \\
\hline Cárnica & 31 & 93 & $85-100$ & & 12 & 75 & $51-100$ & \\
\hline Cría & & & & & 5 & 60 & $17-100$ & \\
\hline
\end{tabular}

Los resultados se expresan como porcentajes y el intervalo de confianza al 95\% (IC 95\%). Prevalencia verdadera (PV).

tener en cuenta es que el ganado Holstein en las granjas de Uruguay ingiere agua de bebederos próximos a las salas de ordeño, mientras que el resto de bovinos lo hace de pozos naturales. Debemos considerar que la infección por $F$. hepatica puede producirse por la ingestión de hierba o agua contaminada (Marcos y col 2007). Recientemente se ha demostrado la presencia de metacercarias de $F$. hepatica en el agua suministrada a granjas de Colombia (Rodríguez y col 2012). Por el contrario, los bovinos Cruce de Uruguay pastan en praderas naturales que se secan durante el verano, lo que probablemente reduzca el riesgo de infección. Al contrario, en Portugal los Cruces alcanzaron las seroprevalencias más elevadas, en coincidencia con lo demostrado en un área próxima del noroeste de España (Sánchez-Andrade y col 2002), posiblemente porque en los pastos se mantienen humedales durante todo el año, y los animales destinados a la producción de carne rara vez se desparasitan (Arias y col 2010).

El control de la fasciolosis radica en protocolos de desparasitación. En la estimación de la prevalencia basada en la detección de anticuerpos debemos tener en cuenta que los hospedadores son tratados con fármacos antiparasitarios. Aunque los valores de anticuerpos se reducen significativamente en los rumiantes que reciben albendazol o netobimin, no se llegan a registrar valores correspondientes a animales no infectados (Arias y col 2009). En ambos países, el ganado vacuno recibe dos dosis de tratamiento fasciolicida, en primavera y en otoño. En Uruguay, solo los animales menores de tres años reciben dos dosis de nitroxinil por año. No se han observado diferencias en cuanto al sexo de los animales, pero en Uruguay los toros rara vez son desparasitados y las vacas Holstein solo pueden ser tratadas durante el período de secado.

Los datos recogidos en el presente trabajo señalan que el ganado vacuno en extensivo tiene un riesgo importante de exposición a $F$. hepatica, especialmente en zonas con clima subtropical húmedo. La elevada seroprevalencia de los terneros, con independencia de las condiciones climáticas, indica que desde que comienzan el pastoreo los animales ingieren metacercarias, por lo que resulta necesario reducir la contaminación de los pastos por metacercarias de $F$. hepatica, debido a que la desparasitación proporciona solo una solución a corto plazo, y la construcción de cercas para evitar que el ganado acceda a las áreas húmedas es una opción rara vez contemplada. En los últimos años se han descrito algunos procedimientos prometedores de control biológico basados en la utilización de hongos saprófitos con actividad ovicida (Dias y col 2012, Arias y col 2013). Con la destrucción de los huevos de $F$. hepatica se prevendría la infección de los caracoles limneidos y por tanto la 
emisión de cercarias. Existen investigaciones en marcha para establecer la forma adecuada que permita añadir estos hongos a las heces de los animales en pastoreo.

\section{REFERENCIAS}

Alcaíno H, W Apt. 1989. Algunos antecedentes sobre la fasciolosis animal y humana. Monografías Medicina Veterinaria 11, 14-29.

Arias MS, JL Suárez, GV Hillyer, I Francisco, E Calvo, R SánchezAndrade, P Díaz, R Francisco, P Díez-Baños, P Morrondo, A Paz-Silva. 2009. A recombinant-based ELISA evaluating the efficacy of netobimin and albendazole in ruminants with naturally acquired fascioliasis. Vet $J 182,73-78$.

Arias M, P Piñeiro, GV Hillyer, JL Suárez, I Francisco, FJ Cortiñas, P Díez-Baños, P Morrondo, R Sánchez-Andrade, A Paz-Silva. 2010. An approach of the laboratory to the field: assessment of the influence of cattle management on the seroprevalence of fascioliasis by using polyclonal- and recombinant-based ELISAs. J Parasitol 96, 626-631.

Arias M, C Lomba, V Dacal, L Vázquez, J Pedreira, I Francisco, P Piñeiro, CF Cazapal-Monteiro, JL Suárez, P Díez-Baños, P Morrondo, R Sánchez-Andrade, A Paz-Silva. 2011. Prevalence of mixed trematode infections in an abattoir receiving cattle from northern Portugal and north-west Spain. Vet Rec 168, 408-413.

Arias M, CF Cazapal-Monteiro, J Suárez, S Miguélez, I Francisco, JL Suárez, A Paz-Silva, R Sánchez-Andrade, P Mendoza de Gives. 2013. A preliminary analysis of the mixed production of spores of filamentous fungi for preventing soil-transmitted helminthzoonoses. Biomed Res Int 567876. doi: 10.1155/2013/567876.

Bennema SC, E Ducheyne, J Vercruysse, E Claerebout, G Hendrickx, J Charlier. 2011. Relative importance of management, meteorological and environmental factors in the spatial distribution of Fasciola hepatica in dairy cattle in a temperate climate zone. Int J Parasitol 41, 225-233.

Carámbula M. 1996. Pasturas naturales mejoradas. Editorial Hemisferio Sur, Montevideo, Uruguay.

Carnevale S, MI Rodríguez, EA Guarnera, C Carmona, T Tanos, SO Ángel. 2001. Immunodiagnosis of fasciolosis using recombinant procathepsin L cystein proteinase. Diagn Microbiol Infect Dis 41, 43-49.

Castro L, G Brito. 2008. Auditoría de Calidad de Carne Vacuna 2007-2008. $5^{\circ}$ Congreso de Producción, Industrialización y Comercialización de Carne "Del Campo al Plato", Instituto Nacional de Carne, Montevideo, Uruguay.

Chauvin A, G Bouvet, C Boulard. 1995. Humoral and cellular immune responses to Fasciola hepatica experimental primary and secondary infection in sheep. Int J Parasitol 25, 1227-1241.

Conceição MA, RM Durão, IM Costa, JM da Costa. 2002. Evaluation of a simple sedimentation method (modified McMaster) for diagnosis of bovine fascioliosis. Vet Parasitol 105, 337-343.

Conceição MA, RM Durão, IM Costa, A Castro, AC Louzã, JC Costa. 2004. Herd-level seroprevalence of fasciolosis in cattle in north central Portugal. Vet Parasitol 123, 93-103.

Cornelissen JB, CP Gaasenbeek, FH Borgsteede, WG Holland, MM Harmsen, WJ Boersma. 2001. Early immunodiagnosis of fasciolosis in ruminants using recombinant Fasciola hepatica cathepsin L-like protease. Int J Parasitol 31, 728-737.

Dias AS, JV Araújo, FR Braga, JM Araújo, AC Puppin, FM Fernandes, RF Ramos, RM Bertonceli, RG da Silva, WR Perboni. 2012. Biological control of Fasciola hepatica eggs with the Pochonia chlamydosporia fungus after passing through the cattle gastrointestinal tract. Parasitol Res 110, 663-667.

Dunkel AM, MC Rognlie, GR Johnson, SE Knapp. 1996. Distribution of potential intermediate hosts for Fasciola hepatica and Fascioloides magna in Montana, USA. Vet Parasitol 62, 63-70.

Eleftheratos K, G Tselioudis, C Zerefos, P Nastos, C Douvis, I Kapsomenakis. 2011. Observed and predicted climate changes in Uruguay and adjacent areas. Hell J Geosci 45, 83-90.
Fox NJ, PC White, CJ McClean, G Marion, A Evans, MR Hutchings. 2011. Predicting impacts of climate change on Fasciola hepatica risk. PLoS One 6, e16126.

Greiner M, IA Gardner. 2000. Application of diagnostic tests in veterinary epidemiologic studies. Prev Vet Med 45, 43-59.

Hope Cawdery MJ. 1984. Review of economic importance of fascioliasis in sheep and cattle. Irish Vet 3, 14-22.

Ibarra F, N Montenegro, Y Vera, CH Boulard, H Quiroz, J Flores, P Ochoa. 1998. Comparison of three ELISA tests for seropidemiology of bovine fasciolosis. Vet Parasitol 77, 229-236.

INE. 2012. Instituto Nacional de Estatística 2011. Lisboa, Portugal.

Kottek M, J Grieser, C Beck, B Rudolf, F Rubel. 2006. World map of the Köppen-Geiger climate classification updated. Meteorol Z 15, 259-263.

Kuchai JA, MZ Chishti, MM Zaki, MAD Muzaffar Rasool, J Ahmad, H Tak. 2011. Epidemiological aspects of fascioliasis among cattle of Ladakh. Global Vet 7, 342-346.

Kuerpick B, C Fiedor, G von Samson-Himmelstjerna, T Schnieder, C Strube. 2012. Bulk milk-estimated seroprevalence of Fasciola hepatica in dairy herds and collecting of risk factor data in East Frisia, northern Germany. Berl Munch Tierarztl 125, 345-350.

Luzón-Peña M, FA Rojo-Vázquez, M Gómez-Bautista. 1995. Seasonal availability of Fasciola hepatica metacercariae in a temperate Mediterranean area (Madrid, Spain). Zbl Vet Med B 42, 577-585.

MAFF, Ministry of Agriculture, Fisheries and Food. 1986. Manual of veterinary parasithological laboratory techniques. HMSO, London, UK.

Marcos LA, A Terashima, G Leguía, M Canales, JR Espinoza, E Gotuzzo. 2007. Fasciola hepatica infection in Peru: An emergent disease. Rev Gastroenterol Perú 27, 389-396.

Marley SE, RM Corwin, DP Hutcheson. 1996. Effect of Fasciola hepatica on productivity of beef steers from pasture through feedlot. Agri-Practice 17, 18-23.

Mezo M, M González-Warleta, JA Castro-Hermida, L Muiño, FM Ubeira. 2011. Association between anti-F. hepatica antibody levels in milk and production losses in dairy cows. Vet Parasitol 180, 237-242.

Nari A, H Cardozo, MA Solari, C Petraccia, D Acosta. 1986. Estudio preliminar sobre el desarrollo de Limnaea viatrix D'Orbigny (1835) en condiciones controladas de temperatura y humedad. Veterinaria $22,13-17$.

Oldham G. 1982. Antibody responses to Fasciola hepatica antigens during liver fluke infection of cattle. Curr Top Vet Med Anim Sci 22, 57-82.

OMS, Organización Mundial de la Salud. 1997. Vector control. Methods for use by individuals and communities. World Health Organization, Geneva, Switzerland, Pp 337-356.

Otranto D, D Traversa. 2003. Dicrocoeliosis of ruminants: a little known fluke disease. Trends Parasitol 19, 12-15.

Paz-Silva A, R Sánchez-Andrade, JL Suárez, J Pedreira, M Arias, C López, R Panadero, P Díaz, P Díez-Baños, P Morrondo. 2003. Prevalence of natural ovine fasciolosis shown by demostrating the presence of serum circulating antigens. Parasitol Res 91, 328-331.

Paz-Silva A, GV Hillyer, R Sánchez-Andrade, JR Rodríguez-Medina, M Arias, P Morrondo, P Díez-Baños. 2005. Isolation, identification and expression of a Fasciola hepatica cDNA encoding a 2.9-kDa recombinant protein for the diagnosis of ovine fasciolosis. Parasitol Res 95, 129-135.

Paz-Silva A, GV Hillyer, MS Arias, R Sánchez-Andrade, J Pedreira, JL Suárez, C Lomba, P Díaz, I Francisco, P Díez-Baños, P Morrondo. 2007. A cross-sectional study of fascioliasis in autochthonous cattle from NW Spain by using a 2.9-kDa recombinant protein. Int J Appl Res Vet M 5, 52-56.

Rapsch C, G Schweizer, F Grimm, L Kohler, C Bauer, P Deplazes, U Braun, PR Torgerson. 2006. Estimating the true prevalence of Fasciola hepatica in cattle slaughtered in Switzerland in the absence of an absolute diagnostic test. Int J Parasitol 36, 1153-1158.

Reid JFS, JD Dargie. 1995. Como os estágios adultos de Fasciola hepatica afetam a saúde e a produtividade do bovino. Hora Vet 15, 23-26.

Rodríguez DC, N Pino, G Peñuela. 2012. Microbiological quality indicators in waters of dairy farms: detection of pathogens by PCR in real time. Sci Total Environ 427-428, 314-318. 
Rojo FA, I Ferre. 1999. Parasitosis hepáticas: Fasciolosis. En: Cordero del Campillo M, Rojo Vásquez FA (eds). Parasitología Veterinaria. McGraw-Hill-Interamericana, Madrid, España, Pp 260-272.

Salimi-Bejestani MR, P Cripps, DJ Williams. 2008. Evaluation of an ELISA to assess the intensity of Fasciola hepatica infection in cattle. Vet Rec 162, 109-111.

Sánchez-Andrade R, A Paz-Silva, JL Suárez, R Panadero, P Díez.-Baños, P Morrondo. 2000. Use of a sandwich-enzyme-linked immunosorbent assay (SEA) for the diagnosis of natural Fasciola hepatica infection in cattle from Galicia (NW Spain). Vet Parasitol 93, 39-46.

Sánchez-Andrade R, A Paz-Silva, JL Suárez, R Panadero, J Pedreira, C López, P Díez-Baños, P Morrondo. 2002. Influence of age and breed on natural bovine fasciolosis in an endemic area (Galicia, NW Spain). Vet Res Commun 26, 361-370.
Sanchís J, S Miguélez, MA Solari, P Piñeiro, MI Macchi, G Maldini, J Venzal, P Morrondo, P Díez-Baños, R Sánchez-Andrade, A Paz-Silva, MS Arias. 2011. Seroprevalencia de la fasciolosis bovina en el departamento de Salto (Uruguay). Rev Ibero-Latin Parasitol 70, 163-171.

Sariözkan S, C Yalçin. 2011. Estimating the total cost of bovine fasciolosis in Turkey. Ann Trop Med Parasitol 105, 439-444.

Schweizer G, U Braun, P Deplazes, PR Torgerson. 2005. Estimating the financial losses due to bovine fasciolosis in Switzerland. Vet Rec 157, 188-193.

Sen M, A Yildirim, Z Bişkin, O Düzlü, A Inci. 2011. The investigation of fasciolosis in cattle by copro-ELISA and stool examination techniques around the Derinkuyu region. Türkiye Parazitol Derg 35, 81-85.

Thrusfield M. 2005. Veterinary epidemiology. $3^{\text {rd }}$ ed. Blackwell, Oxford, UK. 